\title{
Fixation Probabilities of Evolutionary Graphs Based on the Positions of New Appearing Mutants
}

\author{
Pei-ai Zhang \\ Department of Mathematics, Jinan University, Guangzhou City 510632, China \\ Correspondence should be addressed to Pei-ai Zhang; qzhzhang@163.com
}

Received 5 December 2013; Accepted 27 January 2014; Published 17 March 2014

Academic Editor: Pu-yan Nie

Copyright (C) 2014 Pei-ai Zhang. This is an open access article distributed under the Creative Commons Attribution License, which permits unrestricted use, distribution, and reproduction in any medium, provided the original work is properly cited.

\begin{abstract}
Evolutionary graph theory is a nice measure to implement evolutionary dynamics on spatial structures of populations. To calculate the fixation probability is usually regarded as a Markov chain process, which is affected by the number of the individuals, the fitness of the mutant, the game strategy, and the structure of the population. However the position of the new mutant is important to its fixation probability. Here the position of the new mutant is laid emphasis on. The method is put forward to calculate the fixation probability of an evolutionary graph (EG) of single level. Then for a class of bilevel EGs, their fixation probabilities are calculated and some propositions are discussed. The conclusion is obtained showing that the bilevel EG is more stable than the corresponding one-rooted EG.
\end{abstract}

\section{Introduction}

The idea of evolution can be dated back to the times of Darwin. In recent years, there is a growing interest in the investigation of evolutionary dynamics on spatial structures of populations; see Lieberman et al. [1], Ohtsuki et al. [2], Nowak et al. (2006), Nie and Zhang [3-5], Shakarian et al. [6], Broom et al. [7], Fu et al. [8], and the references mentioned therein. Evolutionary graph theory is a nice measure to implement evolutionary dynamics, in which evolutionary dynamics are arranged on graphs. Individuals in a population are posed on a graph, the weighted edges denoting reproductive rates which govern how often individuals place offspring into adjacent vertices. When a mutant appears in this population, its fixation probability is the probability that this mutant takes over the whole population. After a mutant appears in a population, this mutant may choose to leave the group. The fixation probability of an EG can be affected by the number of the individuals, the fitness of the mutant, the game strategy, and the structure of the population, where the fitness reflects the fit degree of the mutant. A population with lower fixation probability is consequently more stable than a population with higher fixation probability. The stability of a graph refers to the degree of stabilization. Calculating the fixation probability of an EG is a basic and key task. Many researchers have studied the problem. The evolution dynamics on an EG are treated as a discrete time Markov chain process. Some EGs are sensitive to the positions where the new mutants appear. Some papers as Lieberman et al. [1], Shakarian et al. [6], Nowak et al. (2006), Nie [3], and Zhang et al. [9, 10] have noticed that the positions of the new appeared mutants in EGs are important to their fixation probabilities. For the directed line, only the mutant appearing at the origin vertex can take over the whole population. The fixation probabilities of some multilevel EGs depend on the appearance of the levels of the new mutants. In this paper, we lay emphasis on the position of the new arisen mutant and put forward a way to calculate the fixation probability of an EG and calculate them for the directed lines, directed circles, and a class of bilevel EGs which is discussed by Zhang et al. [9] and correct their results.

The paper is arranged as follows. Section 2 puts forward the method to calculate the fixation probabilities of singlelevel EGs and applies it to directed lines and directed circles. Section 3 gives the method to calculate the fixation probabilities of a kind of bilevel EGs. Section 4 is the conclusion. 


\section{The Fixation Probabilities of Single-Level EGs}

The evolutionary graph was initially introduced in 2005 by Lieberman et al. [1] and significantly developed by Nie and Zhang [3-5], Zhang et al. [9, 10], Ohtsuki et al. [2], and Shakarian et al. [6] and the references therein. In this framework, the structure of a population is modeled by a weighted digraph on vertices $1,2, \ldots, N$. It is supposed that every individual of the population occupies a unique vertex on the graph. The fitness of a resident is 1 . When a new mutant with fitness $r$ appears, it may take over the whole population or disappear. The graph can describe the architecture of cells in a multicellular organism and represent spatial structure among animals or plants in an ecosystem. Moreover, graphs can also represent relationships in a social network of humans, which means that the dynamics describe cultural selection and the spread of new inventions and ideas. The digraph will model evolution dynamics. In each iteration, a random individual $i$ is chosen for reproduction with a probability proportional to its fitness, and the resulting offspring will occupy an adjacent vertex $j$ with the probability $w_{i j}$, which represents the weight on edge $i j$, if there are any. $\mathrm{Up}$ to now, the evolution on EGs is regarded as a discrete time Markov chain process. But for some EGs, their fixation probabilities, however, have a close relation to the positions of the new appearing mutants. Here we put forward a method to calculate the fixation probabilities of EGs, with regard to the position of the new mutants.

Theorem 1. A given EG has $n$ vertices named $1,2, \ldots, N$. Suppose all the residents with fitness 1 are identical and a new mutant with fitness $r$ is introduced. Let $\rho^{(i)}$ be the fixation probability of a new mutant which appears at the vertex $i$. Then the fixation probability $\rho$ of the new mutant which appears in the population is

$$
\rho=\frac{1}{N} \sum_{i=1}^{N} \rho^{(i)} .
$$

Proof. Let $Q$ be the event that a new mutant appears in the population and $Q_{i}(i=1,2, \ldots, N)$ the event that the new mutant appears at the position of vertex $i$. So $Q_{1}, Q_{2}, \ldots, Q_{N}$ are inconsistent and the following equation is true:

$$
Q=Q_{1}+Q_{2}+\cdots+Q_{N}
$$

Let $T$ be the event that the new mutant is fixed; then we have the following equations:

$$
\begin{aligned}
\rho= & P(T Q) \\
= & P\left(T\left(Q_{1}+Q_{2}+\cdots+Q_{n}\right)\right) \\
= & P\left(T Q_{1}\right)+P\left(T Q_{2}\right)+\cdots+P\left(T Q_{n}\right) \\
= & P\left(Q_{1}\right) P\left(T \mid Q_{1}\right)+P\left(Q_{2}\right) P\left(T \mid Q_{2}\right) \\
& +\cdots+P\left(Q_{N}\right) P\left(T \mid Q_{N}\right)
\end{aligned}
$$

$$
\begin{aligned}
& =\frac{1}{N}\left(P\left(T \mid Q_{1}\right)+P\left(T \mid Q_{2}\right)+\cdots+P\left(T \mid Q_{N}\right)\right) \\
& =\frac{1}{N} \sum_{i=1}^{N} \rho^{(i)} .
\end{aligned}
$$

Since the new mutant can appear at any vertices of the population, the vertices are inconsistent, and the individuals are treated identically; the events $Q_{1}, Q_{2}, \ldots, Q_{N}$ are inconsistent such that $T Q_{1}, T Q_{2}, \ldots, T Q_{N}$ are inconsistent. Therefore the second equation is satisfied, $\left(Q_{i}\right)=1 / N(i=1,2, \ldots, N)$, and the result is obtained. The proof is complete.

We have given the statement of the calculation of fixation probabilities of EGs. In the following we apply it to the directed lines and directed circles.

Proposition 2. A directed line with $N$ vertices named $1,2, \ldots, N$ is shown as follows:

$$
\begin{gathered}
\circ \longrightarrow \circ \longrightarrow \circ \longrightarrow \cdots \longrightarrow \circ \longrightarrow \circ \\
142 \quad 3-1 \quad N
\end{gathered}
$$

Suppose a new mutant with fitness $r$ is introduced and all the residents are with fitness 1 . Then the fixation probability of the new mutant is $1 / N$.

Proof. It is obvious that the new mutant will occupy the whole population if it appears at position 1; the new mutant will disappear if it appears at any other positions. So we have $\rho^{(1)}=1$ and $\rho^{i}=0, i=2,3, \ldots, n$. From (1) we have

$$
\rho=\frac{1}{N} \sum_{i=1}^{N} \rho^{(i)}=\frac{1}{N} .
$$

The proof is complete.

We have applied Theorem 1 to the directed lines and we have gotten the same result as Nowak [11] did. In the following we will apply Theorem 1 to directed circles.

Proposition 3. A directed circle with $N$ vertices named $1,2, \ldots, N$ is shown in Figure 1. Suppose a new mutant with fitness $r$ is introduced and all the residents are with fitness 1 . Then the fixation probability of the new mutant is $\rho=(1-$ $1 / r) /\left(1-1 / r^{N}\right)$.

Proof. Let state $k$ be the state of $k$ mutants, $x_{k}^{(i)}$ the probability of reaching state $N$ when starting from state $k$ and starting initially from single new mutant at position $i$, and $P_{k, l}^{(i)}$ the probability of moving from state $k$ to state $l, y_{k}^{(i)}=x_{k}^{(i)}-x_{k-1}^{(i)}$ and $\gamma_{k}^{(i)}=P_{k, k-1}^{(i)} / P_{k, k+1}^{(i)}$ for $i=0,1,2, \ldots, N ; k=1,2, \ldots, N-$ 1. It is clear that $x_{0}^{(i)}=0, x_{N}^{(i)}=1$ and the mutants are in a cluster. By direct calculation, we get $P_{k, k-1}^{(i)}=1 /(N-k+k r)$ and $P_{k, k+1}^{(i)}=r /(N-k+k r)$ such that $\gamma_{k}^{(i)}=P_{k, k-1}^{(i)} / P_{k, k+1}^{(i)}=1 / r$. 


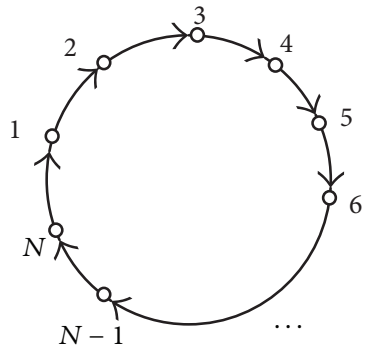

FIGURE 1: A directed circle with $N$ individuals.
From the equation $x^{(i)}=P^{(i)} x^{(i)}$, we have the following equations:

$$
\begin{gathered}
y_{2}^{(i)}=\gamma_{1}^{(i)} x_{1}^{(i)}=\frac{1}{r} x_{1}^{(i)}, \\
y_{3}^{(i)}=\gamma_{1}^{(i)} \gamma_{2}^{(i)} x_{1}^{(i)}=\frac{1}{r^{2}} x_{1}^{(i)}, \ldots, y_{N}^{(i)}=\frac{1}{r^{N-1}} x_{1}^{(i)},
\end{gathered}
$$

is the transition matrix. Since

$$
\begin{aligned}
1 & =x_{N}^{(i)}-x_{0}^{(i)} \\
& =y_{N}^{(i)}+y_{N-1}^{(i)}+\cdots+y_{1}^{(i)} \\
& =\left(1+\frac{1}{r}+\cdots+\frac{1}{r^{N-1}}\right) x_{1}^{(i)},
\end{aligned}
$$

we can obtain the following equations:

$$
\begin{aligned}
\rho^{(i)} & =x_{1}^{(i)}=\frac{1}{1+1 / r+\cdots+1 / r^{N-1}} \\
& =\frac{1-1 / r}{1-1 / r^{N}}, \quad \text { for } i=1,2, \ldots, N .
\end{aligned}
$$

The result of the proposition is therefore obtained from (1) and (9). The proof is complete.

The result in Proposition 3 is the same as that Nowak [11] obtained, but we lay emphasis on the position of the new mutant.

In the section, we gave the calculation of fixation probability which has a close relation with the vertex where the new mutant appears in Theorem 1 and applied it to the directed lines and directed circles. From the above discussion, we can get the conclusion that the directed line cannot be regarded a Markov chain process, for it is sensitive to the position where the new mutant is introduced, while the directed circle can be looked upon as a Markov chain process, for it is insensitive to the position. In the following section, a class of bilevel EGs will be discussed.

\section{The Fixation Probabilities of a Class of Bilevel EGs}

Nie [3] introduced bilevel EGs: the upper levels are stars whereas the lower levels are isothermal EGs. Nie showed that the fixation probabilities of the EGs on two levels are lower than those of the star structure, such that the EGs on two levels are more stable. Nie explained the phenomenon of autoeciousness. Zhang et al. [9] discussed a class of bilevel EGs: the upper levels are isothermal and the lower levels are one rooted. The bilevel EGs reflect hierarchical population structures. The work on bilevel EGs is extended by Zhang et al. [10] where the considered bilevel EGs have different fitness depending on the levels of the graphs. Zhang et al. [10] described several anecdotal applications of bilevel EGs, including the budgerigar of Oceania and the Aptenodytes forsteri (emperor penguins) of Antarctica. Zhou [12] took a step further toward a real-world application by using bilevel EGs and star EGs to examine the stability of various types of business forms. They model corporations with individual decisions (CIDs) as 1-level star graphs and multiperson decision corporations (MDCs) and stock corporations (SCs) are modeled as bilevel graphs. They find that, under reasonable conditions, MDCs have higher fixation probabilities than CIDs, which have higher fixation probabilities than SCs. Hence, through the lens of EGT, SCs represent the most stable organizational structure for business among these three types of business forms. The bilevel EGs and multilevel EGs are important. There are many works to be done in this region. Here we give and correct the fixation probabilities of the bilevel EGs discussed in Zhang et al. [9]. For the bilevel EG as shown in Figure 2, the vertices can be divided into two sets. One is named $A$; the other is named $B$. The vertices in set $A$ are called the leaders with number $m$, while the vertices in set $B$ are called the followers with number $n$. The leaders form an isothermal $E$. When the leaders (set $A$ ) are collapsed into a single vertex, we have a one-rooted EG. In the following theorem, we give the fixation probability of the described bilevel EG.

Theorem 4. For a bilevel EG, the upper level is isothermal with $m$ vertices whereas the lower level is one-rooted with $N$ 


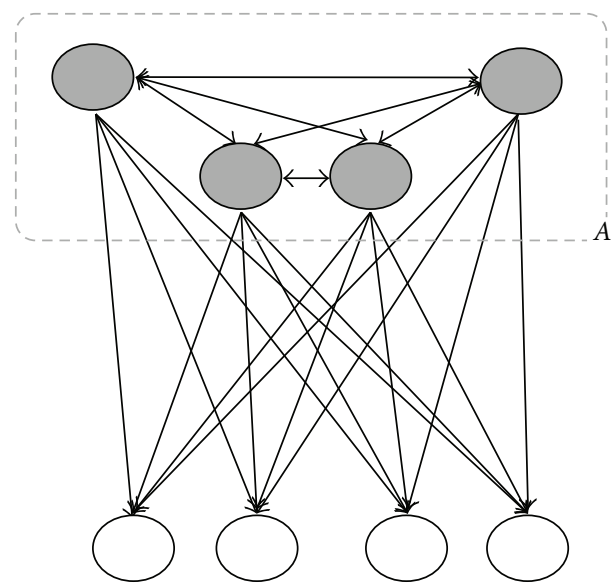

FIgURE 2: A bilevel EG where the leaders (set $A$ ) form an isothermal EG. When they are collapsed into a single vertex, we have a onerooted EG [6].

vertices. If a new mutant with fitness $r$ is introduced and all the residents are with fitness 1 in the EG, its fixation probability is $\rho=(m /(m+N)) \cdot\left(1-r^{-1}\right) /\left(1-r^{-m}\right)$.

Proof. Let $C$ be the event that the new mutant appears in set $A, \bar{C}$ the event that the new mutant appears in set $B$ which is the opposite event of $C, D$ the event that new mutant occupies set $A$, and $E$ the event that the new mutant occupies the whole vertices. If a mutant occupies the whole vertices, it must occupy all the vertices in set $A$. Therefore we have $D \subseteq C$ such that $D=D C$. The fixation probability of a new mutant refers to the probability that the new mutant occupies the whole EG such that $\rho=P(E)$. Since vertices are treated identically, the probability that the new mutant appears in set $A$ is $m /(m+N)$ and the probability that the new mutant appears in set $B$ is $N /(m+N)$. Then we have

$$
\begin{aligned}
\rho & =P(E) \\
& =P((C+\bar{C}) E) \\
& =P(C E+\bar{C} E) \\
& =P(C E)+P(\bar{C} E) \\
& =P(C D E)+P(\bar{C} E) \\
& =P(C) P(D \mid C) P(E \mid C D)+P(\bar{C})(E \mid \bar{C}) \\
& =\frac{m}{m+N} \cdot \frac{1-r^{-1}}{1-r^{-m}} \cdot 1+\frac{N}{m+N} \cdot 0 \\
& =\frac{m}{m+N} \cdot \frac{1-r^{-1}}{1-r^{-m}} .
\end{aligned}
$$

The third equation in the above equations is true for $C$ and $\bar{C}$ are inconsistent such that $C E$ and $\bar{C} E$ are inconsistent. The fourth equation is true for $C D=D$. The fifth equation is true from the probability law. Since the lower level is one rooted, only the new mutant appearing in set $A$ can occupy the whole vertices in set $A$. Therefore, $P(E \mid \bar{C})=0$. The leaders form an isothermal EG, such that $P(D \mid C)=\left(1-r^{-1}\right) /\left(1-r^{-m}\right)$. The proof is complete.

The fixation probability of the bilevel EG is obtained, which corrects the result of Zhang et al. [9]. In the following we will discuss its property.

Proposition 5. Let $\rho$ be the fixation probability of the bilevel $E G$ with $m$ leaders and $N$ followers and let $r$ be the fitness of the new mutant. When all the leaders in the upper level are collapsed into a single vertex, a one-rooted $E G$ with $N+1$ vertices is obtained. The fixation probability of the one-rooted $E G$ with $N+1$ vertices is denoted by $\rho_{1}$. If the new mutant is advantageous, that is, $r>1$, one can get $\rho<\rho_{1}$.

Proof. Since $\rho_{1}$ is the fixation probability of a one-rooted EG with $N+1$ vertices, $\rho_{1}=1 /(N+1)$.

$$
\begin{aligned}
& \text { For } r>1 \\
& \frac{1-r^{-1}}{1-r^{-m}}=\frac{1}{1+1 / r+1 / r^{2}+\cdots+1 / r^{m-1}}<\frac{1}{m}
\end{aligned}
$$

therefore

$$
\rho=\frac{m}{m+N} \cdot \frac{1-r^{-1}}{1-r^{-m}}<\frac{1}{m+N}<\frac{1}{N+1}=\rho_{1} .
$$

The proof is complete.

In this section, we give the fixation probability of a bilevel EG and discuss its proposition. We get the result that the bilevel EG has lower fixation probability than that of the corresponding one-rooted EG when the leaders are collapsed into one vertex. The same conclusion is obtained as that in Nie [3] and Zhang et al. [9].

\section{Conclusion Remark}

In this paper, we lay emphasis on the effect of the position of the new mutant on its fixation probability. We give the fixation probability of an EG of single level. The result is applied to directed lines and directed circles. For a directed line, the fixation probability of a new mutant has a close relation with the position where it appears. Only the mutant appearing at the "first" position will occupy the whole population while the mutant appearing at other positions will disappear. So the directed line is sensitive to the position and the evolutionary dynamics on the directed line cannot be regarded as a Markov chain process. For a directed circle, the fixation probability of the new mutant appearing at each position is identical. So the directed circle is insensitive to the position and the evolutionary dynamics on the directed circle can be regarded as a Markov chain process.

Then the idea is applied to a class of bilevel EGs. The upper levels are isothermal while the lower levels are one rooted. The fixation probabilities are given which correct the result in Zhang et al. [9]. We compare the bilevel EG with the case where all the leaders in the upper level are collapsed into 
only one vertex, that is, the one-rooted EG. For an advantage mutant $r>1$, we get the result that the bilevel EG has lower fixation probability such that the bilevel EG is more stable.

Zhou [12] modeled a CID as a star graph and an MDC as a bilevel EG when the lower level and the upper level are both isothermal. Here we would like to model a CID as a onerooted graph and an MDC as a bilevel EG when the upper level is isothermal and the lower level is one rooted. From the conclusion of Proposition 5 we know that the fixation probability of the bilevel graph is lower than that of the corresponding one-rooted graph such that the bilevel EG is more stable. The result, however, is accordant with Zhou [12].

\section{Conflict of Interests}

The author declares that there is no conflict of interests regarding the publication of this paper.

\section{Acknowledgment}

This work is supported by the Project of Humanities and Social Sciences of Ministry of Education of China (no. 11YJAZH118).

\section{References}

[1] E. Lieberman, C. Hauert, and M. A. Howak, "Evolutionary dynamics on graphs," Nature, vol. 433, no. 7023, pp. 312-316, 2005.

[2] H. Ohtsuki, C. Hauert, E. Lieberman, and M. A. Nowak, "A simple rule for the evolution of cooperation on graphs and social networks," Nature, vol. 441, no. 7092, pp. 502-505, 2006.

[3] P.-Y. Nie, "Evolutionary graphs on two levels," Ars Combinatoria, vol. 86, pp. 115-120, 2008.

[4] P.-Y. Nie and P.-A. Zhang, "Fixation time for evolutionary graphs," International Journal of Modern Physics B, vol. 24, no. 27, pp. 5285-5293, 2010

[5] P.-Y. Nie and P.-A. Zhang, "Evolitopmary graphs with frequency dependent fitness," International Journal of Modern Physics B, vol. 23, no. 4, pp. 537-543, 2009.

[6] P. Shakarian, P. Roos, and A. Johnson, "A review of evolutionary graph theory with applications to game theory," BioSystems, vol. 107, no. 2, pp. 66-80, 2012.

[7] M. Broom, C. Hadjichrysanthou, and J. Rychtáŕ, "Evolutionary games on graphs and the speed of the evolutionary process," Proceedings of the Royal Society A, vol. 466, no. 2117, pp. 13271346, 2010.

[8] F. Fu, L. Wang, M. A. Nowak, and C. Hauert, "Evolutionary dynamics on graphs: efficient method for weak selection," Physical Review E, vol. 79, no. 4, Article ID 046707, 10 pages, 2009.

[9] P.-A. Zhang, P.-Y. Nie, D.-Q. Hu, and F.-Y. Zou, "The analysis of bi-level evolutionary graphs," BioSystems, vol. 90, no. 3, pp. 897-902, 2007.

[10] P.-A. Zhang, P.-Y. Nie, and D.-Q. Hu, "Bi-level evolutionary graphs with multi-fitness," IET Systems Biology, vol. 4, no. 1, pp. 33-38, 2010.

[11] M. A. Nowak, Evolutionary Dynamics Exploring the Equations of Life, Harvard University Press, Cambridge, Mass, USA, 2005.
[12] A. Zhou, "Stability analysis for various business forms", in Applied Economics, Business and Development, Q. Zhou, Ed., vol. 208 of Communications in Computer and Information Science, pp. 1-7, Springer, Berlin, Germany, 2011. 


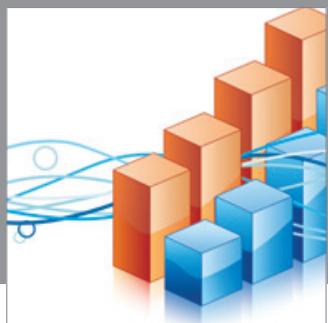

Advances in

Operations Research

mansans

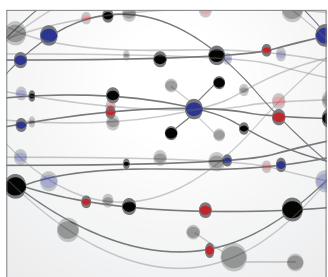

The Scientific World Journal
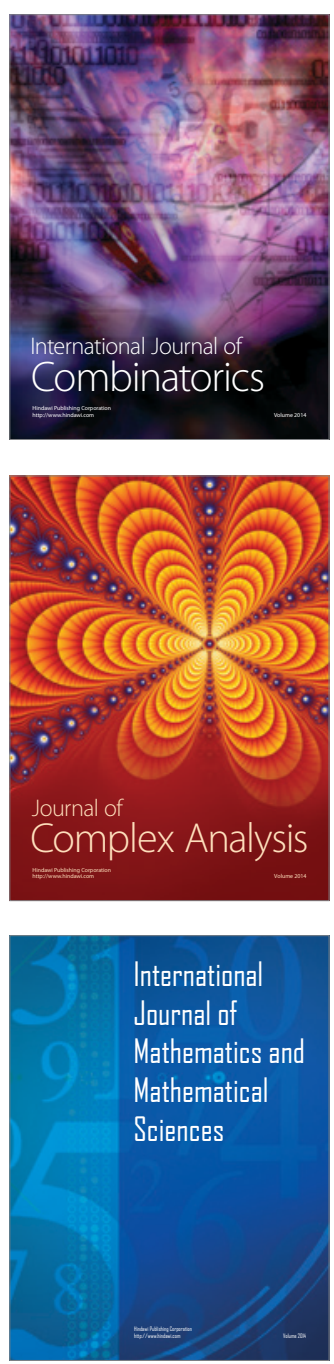
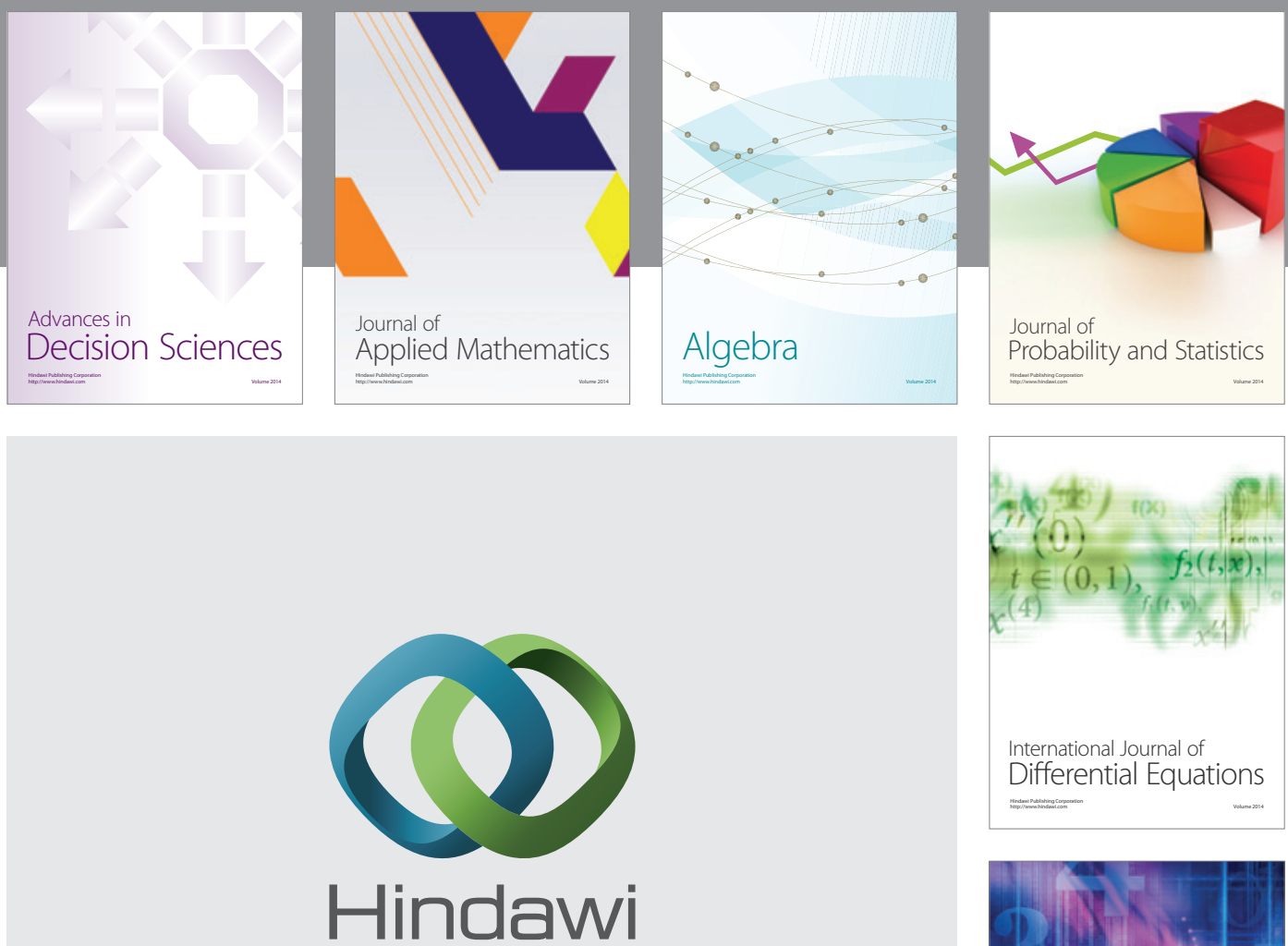

Submit your manuscripts at http://www.hindawi.com
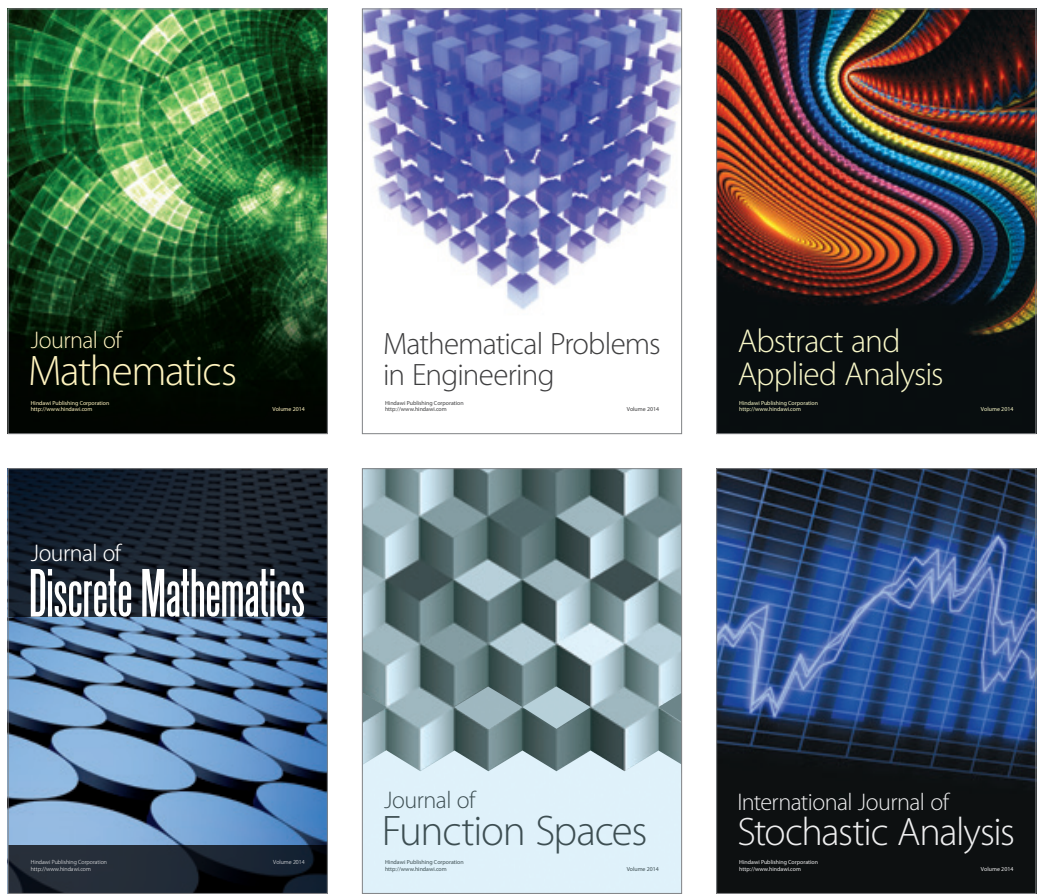

Journal of

Function Spaces

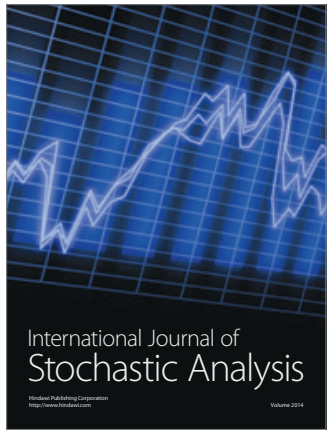

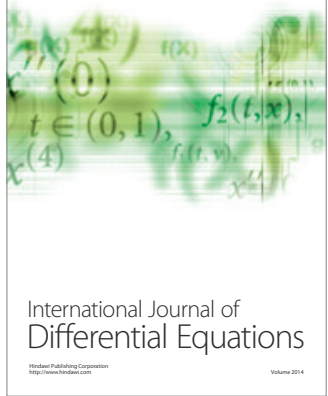
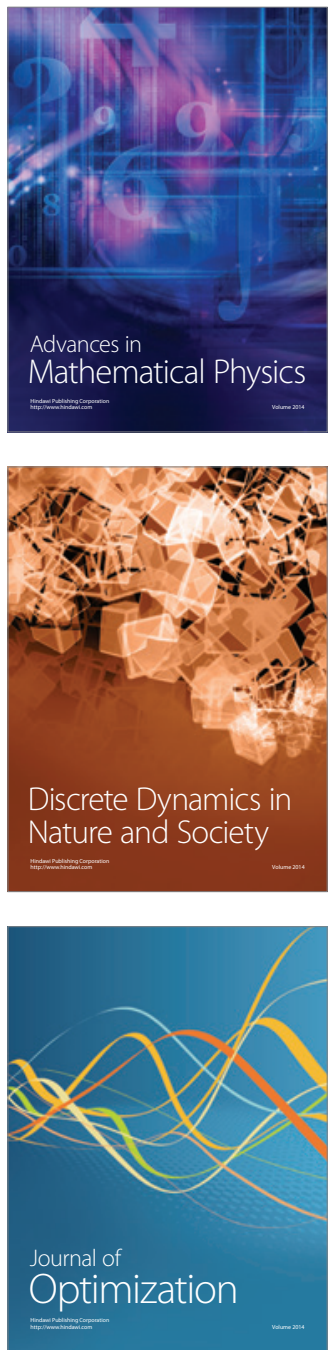